\title{
Characterization of a waveguide Mach-Zehnder interferometer using PDMS as a cover layer
}

\author{
S. M. Lindecrantz \\ susanlindecrantz@gmail.com \\ J.-C. Tinguely \\ B. Singh Ahluwalia \\ O. G. Hellesø
}

\author{
UiT - The Arctic University of Norway, Department of Physics and Technology, N-9037 Tromsø, \\ Norway \\ UiT - The Arctic University of Norway, Department of Physics and Technology, N-9037 Tromsø, \\ Norway \\ UiT - The Arctic University of Norway, Department of Physics and Technology, N-9037 Tromsø, \\ Norway \\ UiT - The Arctic University of Norway, Department of Physics and Technology, N-9037 Tromsø, \\ Norway
}

A Mach-Zehnder interferometer made with shallow rib waveguides is studied experimentally and using simulations. The rib-height giving single-mode guidance is found as function of core thickness and polarization. Devices have been made using shallow rib waveguides ( $5 \mathrm{~nm}$ rib height) in silicon nitride. The sensitivity and the limit of detection (LOD) is studied experimentally regarding the length of the sensing window and for two cover media: water with hydrochloric acid $(\mathrm{HCl})$ and polydimethylsiloxane (PDMS). For $\mathrm{HCl}$-solution, which is the standard method for testing Mach-Zehnder interferometers, the measured sensitivity and LOD was $13000 \pi \mathrm{rad} / \mathrm{RIU}$ and $1.1 \times 10^{-7} \pi$ RIU, respectively, for a $2 \mathrm{~cm}$ long sensing window. This is comparable to the best results reported previously. With PDMS as the cover medium, the temperature of the device was increased in order to measure the sensitivity. This is a new approach that makes it possible to measure the sensitivity with a solid cover medium which has a relatively high temperature coefficient for the refractive index. Measured sensitivity and LOD was $15200 \pi \mathrm{rad} / \mathrm{RIU}$ and $1.3 \times 10^{-7} \pi$ RIU, respectively, again for a $2 \mathrm{~cm}$ long sensing window. Measured sensitivities agreed with simulations and increased linearly with sensing length as expected. However, the LOD showed a minimum for $2 \mathrm{~cm}$ sensing length. This was mainly due to increased noise for $3 \mathrm{~cm}$ sensing length, both for $\mathrm{HCl}$-solution and PDMS. With higher sensitivity and similar LOD for PDMS compared to $\mathrm{HCl}$-solution, it is concluded that using the temperature dependence of PDMS is a good alternative for testing Mach-Zehnder interferometers.

[DOI: http://dx.doi.org/10.2971/jeos.2015.15020]

Keywords: Rib waveguide, integrated optics, Mach-Zehnder interferometer, polydimethylsiloxane

\section{INTRODUCTION}

Waveguide Mach-Zehnder interferometers are highly sensitive and have been used successfully for a range of applications, e.g. sensing of pressure [1], gases [2], volatile organic compounds [3], DNA/RNA, proteins and other biological molecules[4,5]. Several sensors can be combined on a single chip, giving a compact, sensitive and stable device [5]. The principle of the sensor is to use waveguides to split the light into two arms that recombine after a certain distance, see Figure 1 . One of the arms (the sensing arm) is covered with a sensitive layer capturing the chemically inert species, while the other arm (the reference arm) is covered with a protective cladding. The evanescent field of the sensing arm interacts with the sensitive layer. A local change of refractive index in this region will change the effective refractive index of the guided mode of the sensing arm. When the reference mode and the sensing mode combine, constructive or destructive interference is obtained depending on the phase change. The phase change of the interference signal can be correlated to the concentration of the measurand with prior calibration.

In this article, the sensitivity and limit-of-detection (LOD) is studied and compared using simulations and measurements for water and polydimethylsiloxane (PDMS) as the cover mediums. Adding hydrochloric acid $(\mathrm{HCl})$ to water is the standard method for characterizing waveguide Mach-Zehnder interferometers and the results can be directly compared with the state-of-the-art. A limit of detection of $10^{-7}-10^{-8}$ refractive index units (RIU) have been demonstrated with rib waveguides and with a water-solution as the cover medium $[6,7]$. Biological materials will normally be dissolved in water, and the most common cover media for biosensors thus have refractive indices close to that of water. For other types of sensors, e.g. gas sensors, various solids can be used to absorb the measurand and hence give a change in the refractive index of the solid. As an example, PDMS doped with cryptophane-A can be used to detect methane with surface plasmon resonance [8, 9]. PDMS is an organic material with a relatively high thermo-optic coefficient, and consequently gives a high thermal sensitivity for the guided mode [10]. This will normally give unwanted temperature dependency for the sensor. However, in this work the high thermal sensitivity is used to characterize the 

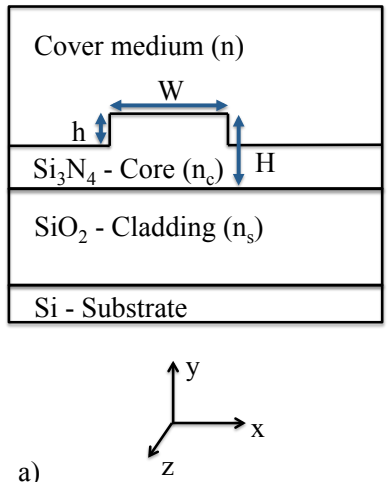

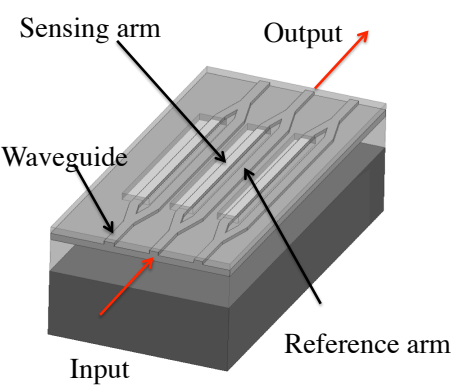

b)
FIG. 1 Shows the outline of the designed sensor with a) cross-section of the waveguide structure and b) top-view of three Mach-Zehnder interferometers on a chip. $W$ is the waveguide width, $H$ is the core thickness and $h$ is the rib height. Note that the drawings are not to scale.

sensor. A PDMS-layer is deposited on the sensing arm of a Mach-Zehnder interferometer, while the reference arm is covered by silica, which has a significantly smaller thermooptic coefficient. By increasing the temperature of the device, the temperature of the PDMS-layer also increases. As the thermo-optic coefficient of PDMS is known, a controlled change in the refractive index of the PDMS-layer is thus obtained and the sensitivity of the sensor to this change can be measured.

Shallow rib waveguides with a $\mathrm{Si}_{3} \mathrm{~N}_{4}$ core are suitable for Mach-Zehnder interferometers due to the high refractive index of $\mathrm{Si}_{3} \mathrm{~N}_{4}$, good transparency in the visible range and the low propagation losses of the shallow rib structure [11, 12]. To obtain high visibility, the waveguides of the interferometer must be single-mode. We use simulations to determine the geometrical parameters of the waveguide cross-section which result in single-mode waveguides. Comsol Multiphysics, which is based on the finite element method, is used to determine single-mode conditions and to simulate the sensitivity of the sensor to changes in the refractive index of the cladding.

\section{SIMULATIONS}

The waveguides used in this work had a guiding core of $\mathrm{Si}_{3} \mathrm{~N}_{4}$ $\left(\mathrm{n}_{c}=2.00\right)$ on a silica layer $\left(2 \mu \mathrm{m}\right.$ thick, $\left.\mathrm{n}_{s}=1.45\right)$. The waveguide width was set to $2 \mu \mathrm{m}$ for the simulations, which can easily be obtained with standard photolithography. Various core thicknesses and rib heights were simulated, as will be shown in the next section. For the cover medium, simulations were done for refractive indices corresponding to water $(n=1.33)$ and to PDMS $(\mathrm{n}=1.412)$. Figure 1 shows the cross-section of the rib waveguide used in the simulation, and the top-view of three Mach-Zehnder interferometers. A wavelength of 785 $\mathrm{nm}$ was used for all the simulations and experiments. Strip waveguides have also been simulated with, by definition, the rib height equal to the core thickness. In order to find the number of modes for the waveguide, and more specifically to find the limit where it becomes single-mode, a 2D mode analysis of the waveguide cross-section was done using Comsol Multiphysics. This mode analysis was also used to find the sensitivity, as explained in the next section. Figure 2 shows examples of the simulated modes for different waveguide widths and polarizations. The evanescent field of a guided mode decreases exponentially away from the waveguide. The single-mode limit was chosen as the point where the evanescent field of the first-order mode ceased to decrease exponentially.

The results obtained with Comsol were compared to simulations performed with Fimmwave (Photon Design, Oxford, UK), which is based on the film mode matching method (FMM) [13]. Using Fimmwave, the single-mode limit was taken as the point where the first-order mode becomes leaky (TE-polarization) or where its loss increases sharply (TMpolarization) when decreasing the rib height [14].

\subsection{Geometrical parameters giving single-mode waveguides}

Figure 3 shows the maximum rib height which will result in single-mode waveguides, as function of the core thickness. The waveguide width is $2 \mu \mathrm{m}$ and the single-mode limit is shown for both TE- and TM-polarization and with water and PDMS as cover media. The results obtained with Comsol were confirmed by Fimmwave (not shown). For both polarizations, the differences between the two methods are small and comparable to the resolution of the methods (approximately $1 \mathrm{~nm})$.

For the core thicknesses considered in Figure 3, the single mode limit is decreasing for TM-polarization and slowly increasing for TE-polarization. As the core thickness decreases towards zero, the guiding of the first-order mode will cease for any rib height. Thus, for decreasing core thickness, the single-mode limit increases. This effect is dominating for TMpolarization in Figure 3. For fixed rib height and increasing core thickness, the ratio of rib height to core thickness decreases. This will, at some point, give weaker guiding and the single-mode limit will increase with core thickness. This effect causes the slow increase of the single-mode limit for TEpolarization. For a given polarization and core thickness, the single-mode limit is approximately the same for water, Figure 3(a), as for PDMS, Figure 3(b).

To make single-mode rib waveguides, it is necessary to have tight control of the etching of the rib because a rib height of less than $7 \mathrm{~nm}$ is required. On the other hand, the width can be relatively large. The width of $2 \mu \mathrm{m}$ considered in these simulations, is compatible with standard photolithography. By reducing the rib height further, towards $3 \mathrm{~nm}$, waveguides can be made single-mode for a width of $4 \mu \mathrm{m}$ [15]. For strip waveguides, the core is etched completely down and the control on the etching process is thus relaxed. However, the width has to be significantly smaller and it is thus difficult to make single-mode strip waveguides with standard contact photolithography.

\subsection{Sensitivity of the sensor}

The evanescent field reaches out into the cover medium on top of the waveguide. Any change of the refractive index of 

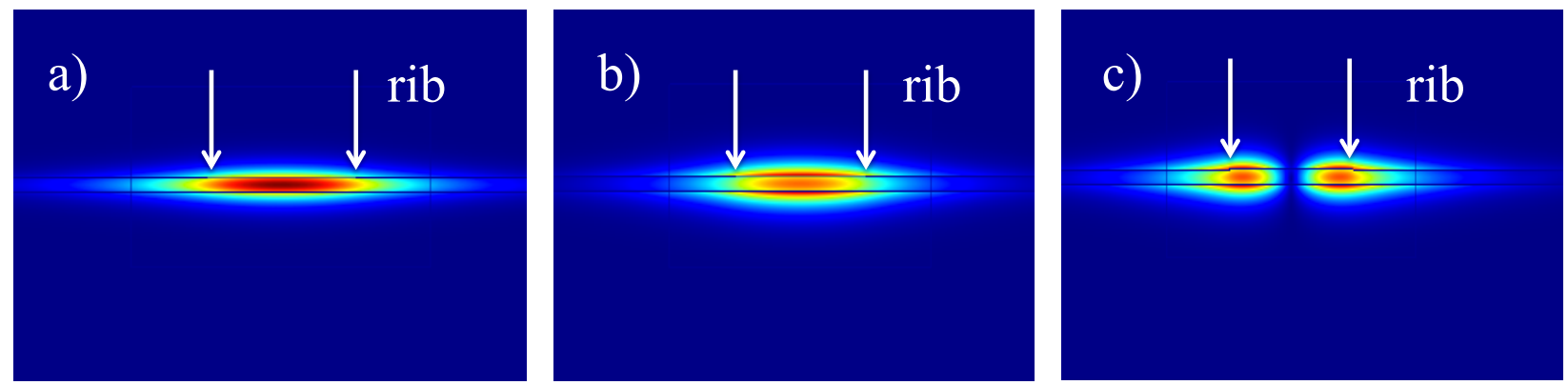

FIG. 2 Examples of modes for a shallow rib waveguide. a) and b) shows the fundamental TE- and TM-mode, respectively ( $5 \mathrm{~nm}$ rib height, $2 \mu \mathrm{m}$ rib width and $200 \mathrm{~nm}$ core thickness). c) shows the first order TM-mode ( $15 \mathrm{~nm}$ rib height, $2 \mu \mathrm{m}$ rib width and $200 \mathrm{~nm}$ core thickness).
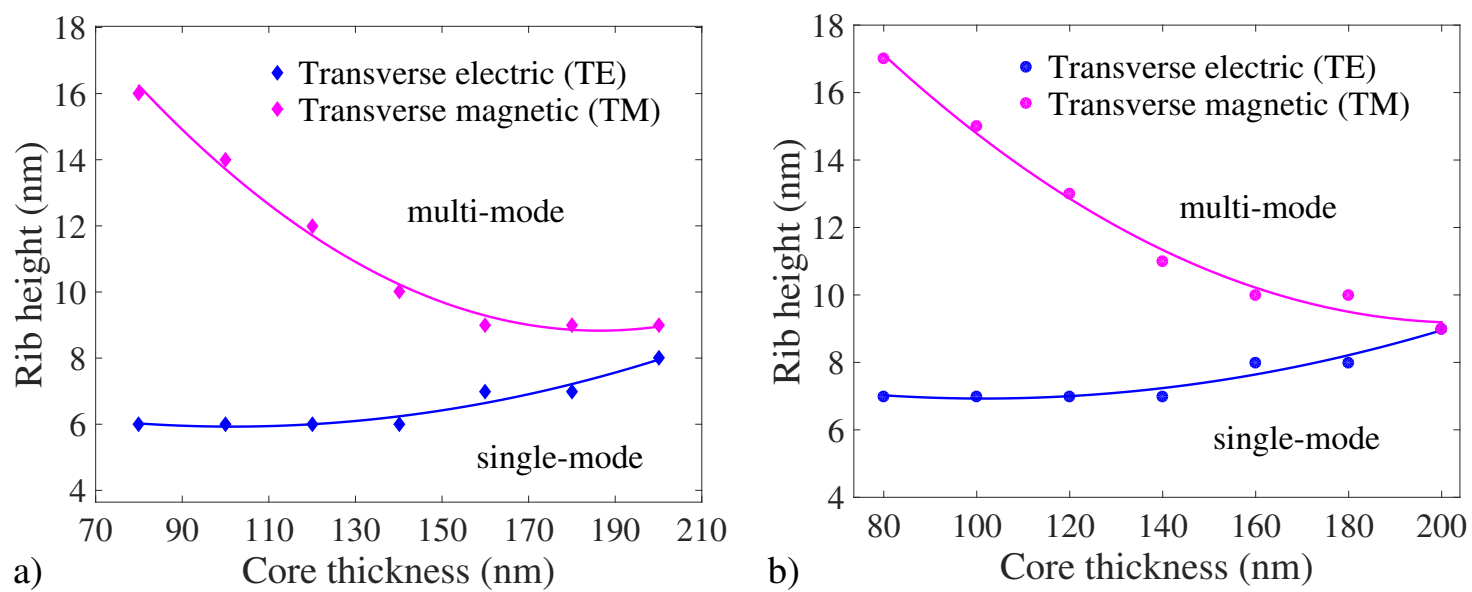

FIG. 3 Shows the simulated single-mode conditions for $2 \mu \mathrm{m}$ wide rib waveguides with TE- and TM-polarization. The single-mode limit is shown for a cover medium of a) water $(n=1.33)$ and $b)$ PDMS $(n=1.412)$.

the sensitive layer will thus change the effective refractive index of the mode. The sensitivity to this change in refractive index is dictated by the waveguide dimensions and by the choice of materials. Homogeneous sensitivity is defined as the rate of change of the effective refractive index of the guided mode, to the rate of change of the refractive index of a homogeneous cover medium. The homogeneous sensitivity can be expressed as [16, 17],

$$
\frac{\Delta N}{\Delta n}=\frac{n}{N} \frac{P_{o}}{P_{T}} *\left(2 \frac{N^{2}}{n^{2}}-1\right)^{r},
$$

where $n$ is the cover medium refractive index, $N$ is the effective refractive index of the guided mode, $P_{O}$ is the power of the guided mode in the cover medium, and $P_{T}$ is the total power of the guided mode. Here $r=0$ for TE-polarization and $r=1$ for TM-polarization. Furthermore, the phase sensitivity for homogeneous sensing can be expressed as:

$$
\frac{\Delta \theta}{\Delta n}=\frac{2 \pi * L}{\lambda_{o}} * \frac{\Delta N}{\Delta n},
$$

where $L$ is the sensing length, $\lambda_{o}$ is the wavelength and $\frac{\Delta N}{\Delta n}$ is the homogeneous sensitivity found in Eq. (1). The minimum detectable phase shift, $\Delta \theta_{\text {min }}$, can be estimated as three times the signal-to-noise ratio, given by the noise, $\sigma$, divided by the maximum intensity change, $\Delta I$, of the fringes:

$$
\Delta \theta_{\min }=\frac{3 \sigma \pi}{\Delta I} .
$$

To compare the performance of various sensors, it is most common to use the limit-of-detection (LOD $\left.=\Delta n_{\text {min }}\right)$, which is given by the minimum detectable phase shift divided by the phase sensitivity:

$$
\Delta n_{\min }=\frac{\Delta \theta_{\min }}{S} .
$$

Simulations were done with Comsol Multiphysics to determine the variables in Eq. (1) for various geometrical parameters, to find the corresponding sensitivity. Figure 4 shows the calculated homogeneous sensitivities as function of the core thickness for strip and rib waveguides, with PDMS and water as the cover media. The sensitivity is very dependent on the core thickness and to some degree on the polarization of the guided light. The sensitivity increases as the core thickness decreases, due to less confinement in the waveguide core, which implies more power in the cover medium. For even less core thickness, the sensitivity decreases as the mode approaches cut-off. In general, maximum sensitivity for TE-polarization is obtained with a thinner waveguide than for TM-polarization. The maximum sensitivity is highest for TM-polarization, being 28\% higher than for TE-polarization for water as the sensing medium. Likewise, for PDMS as the sensing medium, the sensitivity is $18 \%$ higher for TM than for TE-polarization. Regarding water and PDMS as cover medium, the sensitivity is $47 \%$ higher for TE and 35\% higher for TM for PDMS than for water as the cover medium. This can be explained by PDMS having a refractive index closer to that of the waveguide core. If PDMS is used as the sensing medium, it is thus better to test the sensor with PDMS rather than $\mathrm{HCl}$-solution, as the sensitivity is higher for PDMS.

Strip waveguides give approximately $4-8 \%$ higher sensitiv- 

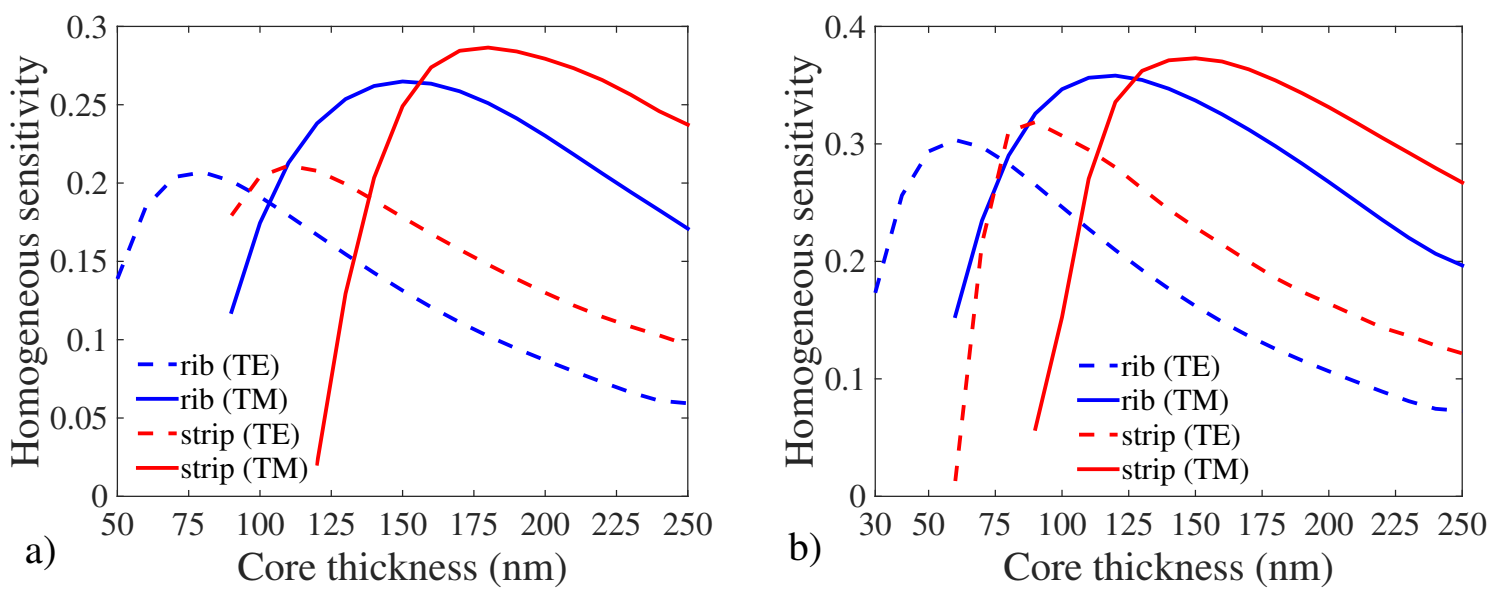

FIG. 4 Shows the homogeneous sensitivity with a) water as cover medium $(n=1.33)$ and b) with PDMS as cover medium ( $n=1.412)$. The sensitivity is shown as function of core thickness for a $0.5 \mu \mathrm{m}$ wide strip waveguide (red) and for a $2 \mu \mathrm{m}$ wide rib waveguide (blue, $5 \mathrm{~nm}$ rib height). Both waveguides are single-mode.

ity compared to rib waveguides. The maximum sensitivity for both polarizations is also shifted towards higher core thicknesses for strip waveguides compared to rib waveguides (180 vs. $150 \mathrm{~nm}$ for water and TM-polarization). The sensitivity is thus not significantly dependent on the choice of strip or rib waveguides. We have also found that the sensitivity does not vary much as function of the waveguide width and rib height, and these results are thus not shown. This can be expected from the small change in effective refractive index of the mode relative to these parameters. Changing the wavelength merely gives a shift of the curves (also not shown).

\section{MEASUREMENT OF THE SENSITIVITY OF THE SENSOR}

Waveguide Mach-Zehnder interferometers were made with a core thickness of $150 \mathrm{~nm}$, rib height of $5 \mathrm{~nm}$ and waveguide widths between $1.5 \mu \mathrm{m}$ and $3 \mu \mathrm{m}$. According to the simulations, see Figure 3 and Figure 4, these dimensions give single-mode waveguides and maximum sensitivity for TMpolarization. The fabrication was done according to Prieto et al. [15]. First, a silica layer with a thickness of $2 \mu \mathrm{m}$ was thermally grown on a silicon wafer. The core layer of silicon nitride, $\mathrm{Si}_{3} \mathrm{~N}_{4}$, was deposited by low-pressure chemical vapor deposition (LPCVD) at $800^{\circ} \mathrm{C}$ and the rib structure of $5 \mathrm{~nm}$ was etched by reactive ion etching (RIE). A protective layer was deposited by plasma-enhanced chemical vapor deposition (PECVD) at $300^{\circ} \mathrm{C}$. Sensing windows were opened in the protective layer by RIE and wet etching. These windows were 1,2 and $3 \mathrm{~cm}$ long, $1 \mu \mathrm{m}$ deep and $50 \mu \mathrm{m}$ wide.

In this section, the sensitivity is measured with water and then with PDMS as the sensing medium (i.e. cover medium). The refractive index of water is increased by adding hydrochloric acid $(\mathrm{HCl})$ and the refractive index of the solution is measured with a refractometer. Thus, changing the cover medium from pure water to an $\mathrm{HCl}$-solution gives interference fringes. By measuring the number of fringes for solutions with various concentrations of $\mathrm{HCl}$, the sensitivity of the sensor is found. For PDMS, a similar approach is not possible. However, the thermo-optic coefficient of the refractive index is $\Delta n / \Delta T=-4.2 \times 10^{-4} \mathrm{RIU} / \mathrm{K}$ for PDMS, compared to $\Delta n / \Delta T=1.28 \times 10^{-5} \mathrm{RIU} / \mathrm{K}$ for silica [18]. Thus, by increasing the temperature of the device, the refractive index of PDMS will decrease and give interference fringes at the output of the interferometer. Note that the reference arm of the interferometer is covered with silica, and it is thus the difference in temperature coefficient between PDMS and silica that gives the interference fringes.

Figure 5 shows the experimental setup. Light from a $785 \mathrm{~nm}$ diode laser was coupled into the waveguide using beam expanding lenses and a $10 x$ objective lens $(\mathrm{NA}=0.30)$. The low magnification, $10 x$, was chosen in order to reduce the influence of thermal and mechanical noise on the input coupling. This was particularly important when increasing the temperature of the waveguide chip. The polarization was rotated with a half-wave plate to obtain TM-polarization for highest sensitivity. A microscope with a CCD camera was used to align the input beam with the waveguide and get optimal end-coupling into the waveguide. A second objective lens $(40 \mathrm{x}, \mathrm{NA}=0.65)$ was used to couple light out from the waveguide and into a photodetector.

For measurements with water as the cover medium, the waveguide chip was enclosed within a microfluidic system connected to an injection port, providing constant feed of water or the diluted $\mathrm{HCl}$. At room temperature, $\mathrm{HCI}$ is a colorless gas that is highly soluble in water. Concentrations of $0.03 \mathrm{~mol} / \mathrm{L}$ to $0.3 \mathrm{~mol} / \mathrm{L}$ of $\mathrm{HCl}$ was added to water. The refractive index of the solution was measured with a refractometer. For example, with a solution of $0.2 \mathrm{~mol} / \mathrm{L} \mathrm{HCI}$, a change in refractive index of 0.0029 RIU relative to water, was obtained.

Water was pumped continuously at a rate of 0.03 to $0.06 \mathrm{ml} / \mathrm{min}$ to the sensing region of the interferometer through the microfluidic system. $5 \mathrm{ml}$ of $\mathrm{HCl}$-solution was injected via an injection port into the flow. Once the injection port was activated, (pure) water was replaced with $\mathrm{HCl}-$ solution, causing the first series of fringes at the output of the interferometer (see the insets in Figure 6). When the volume of $\mathrm{HCl}$-solution was finished, water was pumped back into the system, giving rise to a second series of fringes. After the measurements, the phase was found by counting the fringes, with one fringe equal to $2 \pi$. 


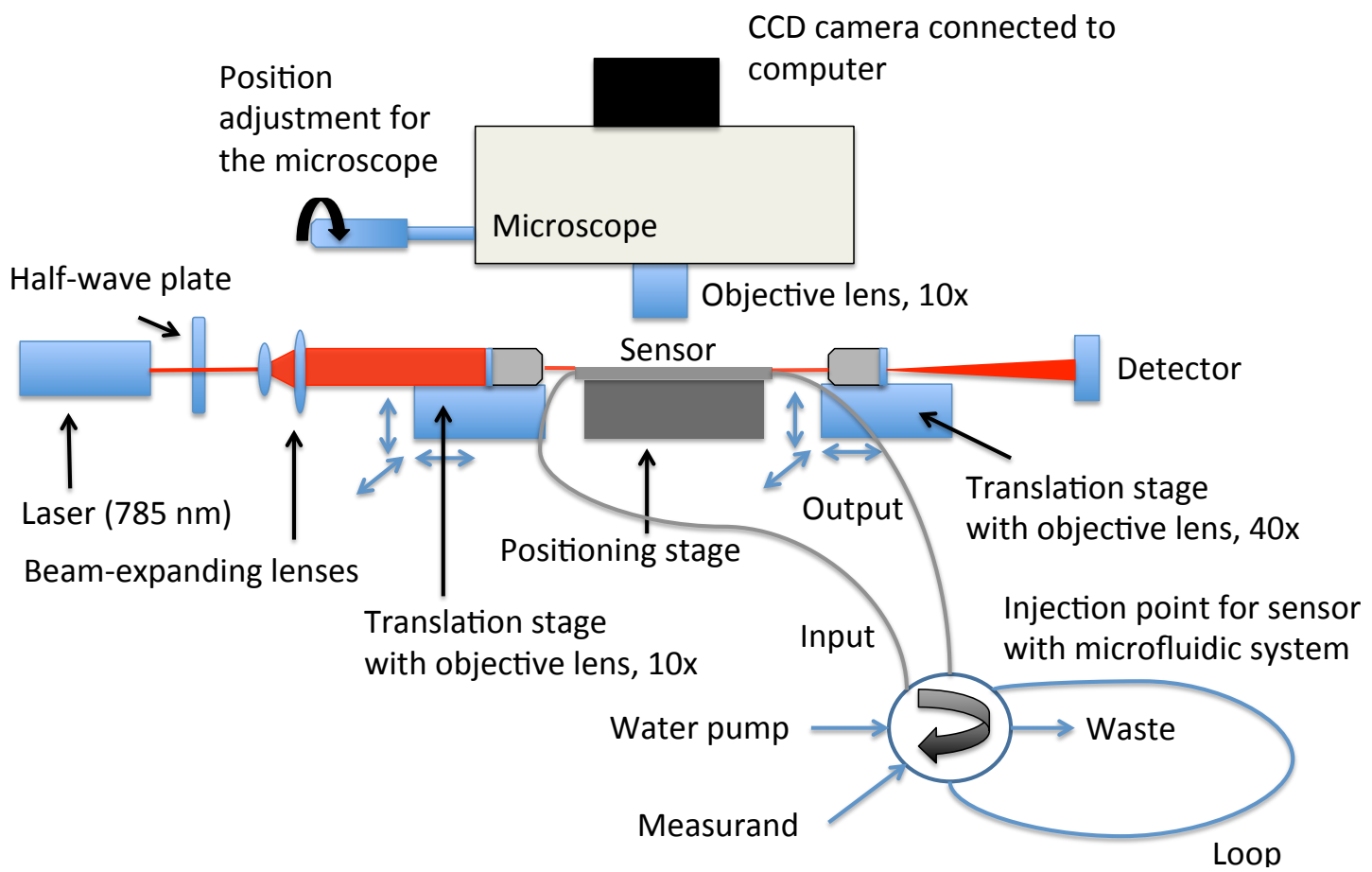

FIC. 5 Shows the experimental setup used for the measurements with a microfluidic system connected to an injection point for $\mathrm{HCl}$ and water pump system.

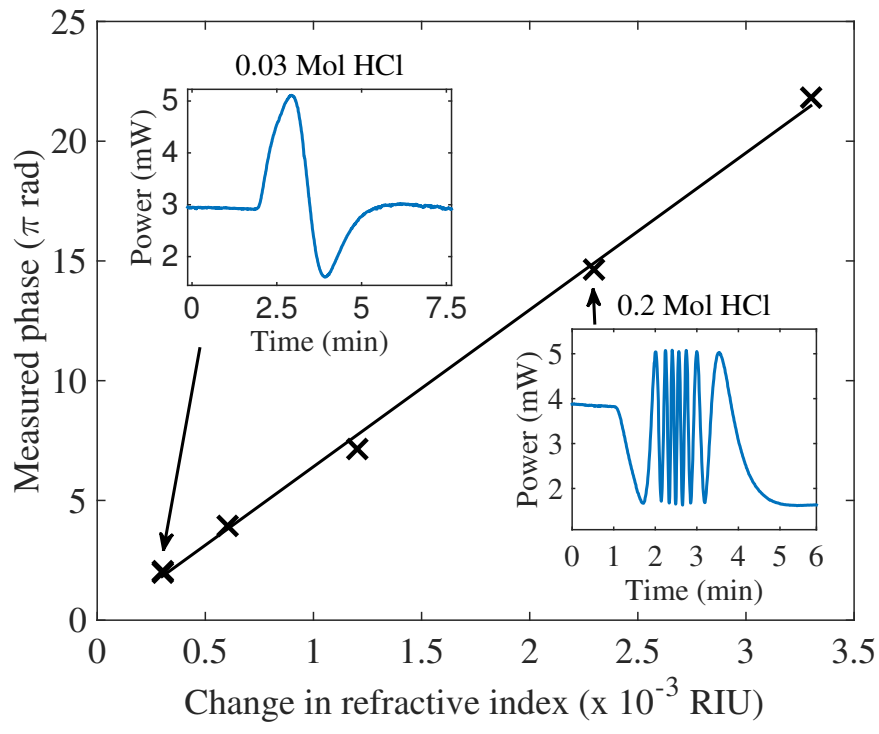

FIG. 6 Shows the calibration curve with $\mathrm{HCl}$-solutions for a Mach-Zehnder interferometer with a $2 \mu \mathrm{m}$ wide waveguide and a sensing length of $1 \mathrm{~cm}$. A straight line is fitted to the measurements and a phase change of $6552 \pi \mathrm{rad} / \mathrm{RIU}$ in water is obtained. The insets show the resulting interference fringes when changing the measurand from pure water to $0.03 \mathrm{~mol}$ and $0.2 \mathrm{~mol} \mathrm{HCl}$, respectively.

Figure 6 shows an example of a calibration curve obtained for different concentrations of $\mathrm{HCI}$ in water. The sensitivity of phase change to change in refractive index of the cover medium is found by making a linear fit to the measured values. This measurement series for $1 \mathrm{~cm}$ sensing length gave a sensitivity of $S=\Delta \theta / \Delta n=6552 \pi \mathrm{rad} / \mathrm{RIU}$ for water as cover medium

To measure the sensitivity with PDMS as the cover medium, the waveguide chip was covered with a layer of PDMS using spin-coating (approximately $25 \mu \mathrm{m}$ thick). A Peltier-element was used to control the temperature, with an aluminium

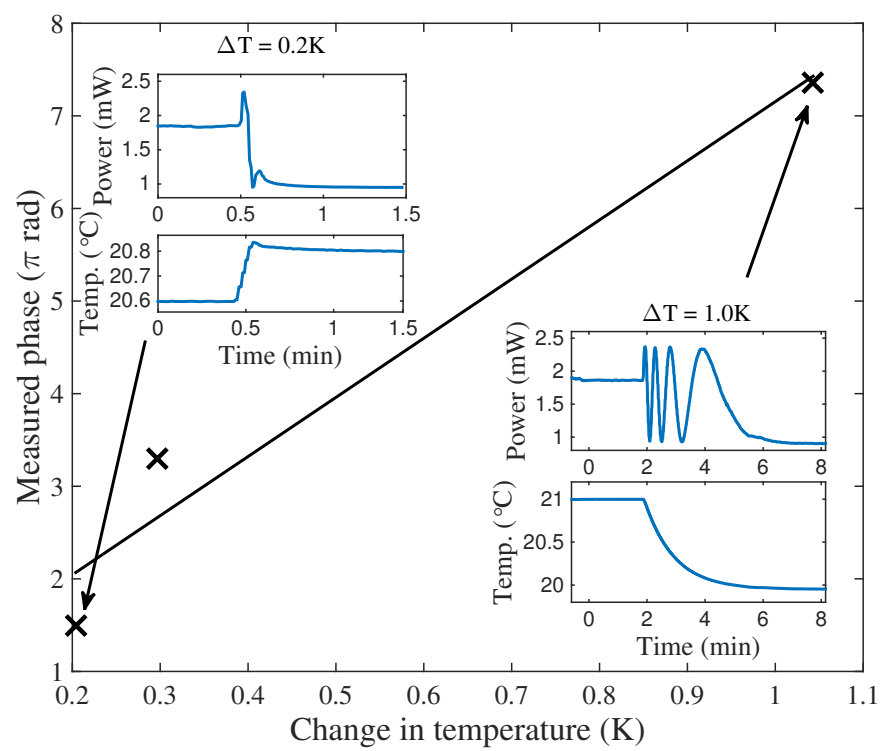

FIC. 7 Shows the calibration curve for a Mach-Zehnder interferometer with PDMS on the sensing arm. The waveguide is $2 \mu \mathrm{m}$ wide and the sensing window is $2 \mathrm{~cm}$ long. A straight line is fitted to the measurements and a phase change of $6.4 \pi \mathrm{rad} / \mathrm{K}$ in PDMS is obtained. The insets show the resulting interference fringes when increasing the temperature by $0.2 \mathrm{~K}$ and $1 \mathrm{~K}$, respectively.

plate on top holding the waveguide chip. A small current was used for the Peltier-element to obtain small temperature changes and avoid oscillations. A thermistor was fixed inside the aluminium plate to precisely measure the temperature changes. Thermal paste was used between the parts to have good thermal conductivity. The temperature and the resulting interference fringes were recorded, as shown in the insets in Figure 7. By applying increasing currents, and thus increasing temperature steps, the sensitivity was measured. An example of a measurement of sensitivity is shown in Figure 7 for an interferometer with a sensing length of $2 \mathrm{~cm}$. 


\begin{tabular}{|l|ccc|ccc|}
\hline Cover medium: & \multicolumn{3}{|c|}{ HCl-solution } & \multicolumn{3}{c|}{ PDMS } \\
\hline$L[\mathrm{~cm}]$ & 1 & 2 & 3 & 1 & 2 & 3 \\
\hline Simulated $S(n)\left[\times 10^{3} \pi \mathrm{rad} / \mathrm{RIU}\right]$ & 6.7 & 13.5 & 20.2 & 8.5 & 17.2 & 25.7 \\
Measured $S(n)\left[\times 10^{3} \pi \mathrm{rad} / \mathrm{RIU}\right]$ & 6.5 & 13.0 & 19.8 & 8.8 & 15.2 & 26.2 \\
Measured $S(T)[\pi \mathrm{rad} / \mathrm{K}]$ & - & - & - & 3.7 & 6.4 & 11.0 \\
$d P[\mathrm{~mW}]$ & 3.4 & 0.6 & 0.8 & 0.7 & 1.4 & 0.7 \\
$\sigma[\mu \mathrm{W}]$ & 7.1 & 0.9 & 9.0 & 1.9 & 2.8 & 4.0 \\
$\Delta \theta_{\min }\left[\times 10^{-3} \pi \mathrm{rad}\right]$ & 6.3 & 4.5 & 33.7 & 8.1 & 6.0 & 17.1 \\
$\mathrm{LOD}(n)\left[\times 10^{-7} \pi \mathrm{RIU}\right]$ & 3.1 & 1.1 & 5.4 & 3.0 & 1.3 & 2.1 \\
$\mathrm{LOD}(T)[\mathrm{mK}]$ & - & - & - & 2.2 & 0.9 & 1.6 \\
\hline
\end{tabular}

TABLE 1 Results obtained for various sensing lengths and with water and PDMS as the cover medium. $L$ is the sensing length, $S$ is the phase sensitivity as function of the refractive index $S(n)$ or temperature $S(T), d P$ is the mean visibility of the fringes, $\sigma$ is the noise of the signal, $\Delta \theta_{\text {min }}$ is the minimum phase-change that can be detected and LOD is the limit-of-detection as function of refractive index, $\operatorname{LOD}(n)$, or temperature, $\operatorname{LOD}(T)$.

This measurement series gave a temperature sensitivity of $S=\Delta \theta / \Delta T=6.4 \pi \mathrm{rad} / \mathrm{K}$. The temperature sensitivity is related to the phase sensitivity by:

$$
\frac{\Delta \theta}{\Delta n}=\frac{\Delta \theta}{\Delta T} \times \frac{\Delta T}{\Delta n}
$$

As previously noted, the thermo-optic coefficient of the refractive index of PDMS is $\Delta n / \Delta T=-4.2 \times 10^{-4} \mathrm{RIU} / \mathrm{K}$ [18]. The phase sensitivity $S$ can thus be found from Eq. (5). For PDMS, the phase sensitivity is thus calculated from the measured temperature sensitivity, while for $\mathrm{HCl}$-solution, the phase sensitivity is measured directly by increasing the refractive index of the $\mathrm{HCl}$-solution.

A summary of the results obtained for various sensing lengths is shown in Table 1 for both HCl-solution and PDMS. The sensitivity increased linearly with the length of the sensing window, as expected, and with very good correspondence with the simulation results. This implies that the thermo-optical coefficient used for PDMS is correct. The sensitivity for PDMS is higher than for $\mathrm{HCl}$-solution due to the higher refractive index (1.42 vs. 1.33), as discussed in the simulation part.

As the sensing length increases, propagation losses are expected to reduce the fringe visibility. However, this effect is not clear from the results in Table 1, indicating that random coupling losses and waveguide defects are more important sources of loss than evenly distributed propagation losses. The noise level increases with sensing length, at least for PDMS. For HCl-solution, the flow cell had a length of $3 \mathrm{~cm}$ and was thus as long as the longest sensing window. The positioning of the flow cell relative to the sensing window and flow effects near the edges of the flow cell might have given increased noise for a sensing length of $3 \mathrm{~cm}$. A longer chip with space for a longer flow cell might give lower noise and a lower detection limit for a $3 \mathrm{~cm}$ sensing length. For PDMS, noise is increasing with sensing length, indicating that temperature noise increases with length and that the sources of the noise are uniformly distributed. The temperature noise of the Peltier-element was approximately $0.5 \mathrm{mK}$. Reducing the temperature noise of the regulation system will also reduce the noise for the optical measurement. Due to the higher noise for $3 \mathrm{~cm}$ sensing length than for $2 \mathrm{~cm}$, for both $\mathrm{HCl}$-solution and PDMS, the limit-of-detection is lowest for a sensing length of $2 \mathrm{~cm}$, at 1.1 and $1.3 \times 10^{-7} \pi$ RIU, respectively.
As shown in Table 1, an LOD for temperature of approximately $1 \mathrm{mK}$ corresponds to an LOD for phase of approximately $10^{-7} \pi$ RIU with PDMS on the sensing arm. Thus it is possible to use the PDMS layer for measuring temperature directly with a limit of detection of approximately $1 \mathrm{mK}$. If using PDMS as a sensitive layer for gas measurement, the temperature stability will influence the LOD for the gas measurement. If temperature stability is the limiting noise source and PDMS is used on the sensing arm only, a temperature noise of $1 \mathrm{mK}$ will thus give an LOD for phase of $1 \times 10^{-7} \pi$ RIU. It might be possible to improve this if the reference arm is also covered with PDMS and subsequently protected with a layer that stops gas diffusion.

\section{CONCLUSION}

The main factors for the sensitivity are the core thickness relative to the wavelength of light, the polarization of the light and the refractive index of the core and cover medium. For shallow rib waveguides and a wavelength of $785 \mathrm{~nm}$, the core thickness giving maximum sensitivity was found to be $80 \mathrm{~nm}$ and $150 \mathrm{~nm}$ for TE- and TM-polarization, respectively, with water as the cover medium. With PDMS as the cover medium, the corresponding values were 60 and $120 \mathrm{~nm}$.

The sensitivity was found to be approximately $28 \%$ higher for TM-polarization than for TE-polarization for water and $18 \%$ higher for TM-polarization than for TE-polarization for PDMS. Strip waveguides give $4-8 \%$ higher sensitivity than rib waveguides. It is possible to make the shallow rib waveguides with standard contact photolithography, significantly simplifying the fabrication process, and the small reduction of sensitivity is thus justified. The sensitivity is $47 \%$ higher for TE and 35\% higher for TM for PDMS than for water as the cover medium. This can be explained by PDMS having a refractive index closer to that of the waveguide core. However, in most cases it will be the type of measurand, e.g. biological or chemical, that decides the choice of cover medium, rather than the higher sensitivity for PDMS.

Sensitivity was measured with $\mathrm{HCl}$ as the cover medium and a Mach-Zehnder interferometer with $2 \mu \mathrm{m}$ waveguide width, $5 \mathrm{~nm}$ rib height, $150 \mathrm{~nm}$ core thickness and 1, 2 and $3 \mathrm{~cm}$ sensing lengths. The measured sensitivities agreed well with the 
simulated values. The lowest limit of detection was found for a sensing length of $2 \mathrm{~cm}$, with $\mathrm{LOD}=1.1 \times 10^{-7} \pi$ RIU. This is comparable to the results obtained in the literature $[7,19]$. By improving the flow cell and reduce the noise for a sensing length of $3 \mathrm{~cm}$, it may be possible to reduce the limit of detection further.

Water is the normal cover medium for biological sensors. However, for gas sensors, it is more common to have a solid cover medium and a different approach must be used when measuring the sensitivity. In this work, we have covered the sensing arm with PDMS and applied a temperature change to the sensor, which decreases the refractive index of the cover layer. This, in turn, was used to measure the sensitivity with PDMS as a cover medium. The sensitivity was, as expected, higher than for $\mathrm{HCl}$-solution and the limit of detection was almost the same, at $1.3 \times 10^{-7} \pi$ RIU. This demonstrates that PDMS can be used to test waveguide Mach-Zehnder interferometers and it is thus an alternative to testing with $\mathrm{HCl}$ solution. We chose to use a low-magnification objective for input coupling (10x), giving a relatively large spot on the endface of the waveguide. This was done in order to reduce noise from the input coupling when increasing the temperature. However, it comes at the expense of reduced coupling. In general, some experimental precautions must be taken when changing the temperature of the chip, as thermal expansion of the mechanical stage can reduce the input coupling.

It is also possible to use the PDMS-layer for temperature measurement, with a limit of detection of approximately $1 \mathrm{mK}$ demonstrated here. If using a PDMS layer for sensing a different measurand, e.g. PDMS doped with cryptophane-A for methane-sensing, the thermal noise must be on the order of 1 $\mathrm{mK}$ to obtain a limit of detection of $1 \times 10^{-7} \pi \mathrm{RIU}$, if PDMS is used only on the sensing arm.

\section{ACKNOWLEDGEMENTS}

This work is part of the project Subsea Sensors for Oil and Gas funded by the Research Council of Norway. The waveguides were made by Carlos Dominguez and co-workers (CSIC, Barcelona, Spain).

\section{References}

[1] C. Wagner, J. Frankenberger, and P. P. Deimel, "Optical pressure sensor based on a Mach-Zehnder interferometer integrated with a lateral a-Si:H p-i-n photodiode," IEEE Photonic. Tech. L. 5, 1257-1259 (1993).

[2] N. Fabricius, G. Gauglitz, and J. Ingenhoff, "A gas sensor based on an integrated optical Mach-Zehnder interferometer," Sensor. Actuat. B-Chem. 7, 672-676 (1992).

[3] A. L. Siarkowski, L. F. Hernandez, B.-H. V. Borges, and N. I. Morimoto, "Sensing based on Mach-Zehnder interferometer and hydrophobic thin films used on volatile organic compounds detection," Opt. Eng. 51, 054401 (2012).

[4] A. Densmore, D.-X. Xu, S. Janz, P. Waldron, J. Lapointe, T. Mischki, G. Lopinski, et al., "Sensitive label-free biomolecular detection using thin silicon waveguides," Adv. Opt. Technol. 2008, 725967 (2008).

[5] P. Kozma, F. Kehl, E. Ehrentreich-Förster, C. Stamm, and F. F. Bier, "Integrated planar optical waveguide interferometer biosensors: a comparative review," Biosens. Bioelectron. 58, 287-307 (2014).

[6] R. Heideman, and P. Lambeck, "Remote opto-chemical sensing with extreme sensitivity: design, fabrication and performance of a pigtailed integrated optical phase-modulated Mach-Zehnder interferometer system," Sensor. Actuat. B-Chem. 61, 100-127 (1999).

[7] S. Dante, D. Duval, B. Sepúlveda, A. B. González-Guerrero, J. R. Sendra, and L. M. Lechuga, "All-optical phase modulation for integrated interferometric biosensors," Opt. Express 20, 7195-7205 (2012).

[8] C. Boulart, M. C. Mowlem, D. P. Connelly, J.-P. Dutasta, and C. R. German, "A novel, low-cost, high performance dissolved methane sensor for aqueous environments," Opt. Express 16, 12607-12617 (2008).

[9] C. Boulart, R. Prien, V. Chavagnac, and J.-P. Dutasta, "Sensing dissolved methane in aquatic environments: an experiment in the central Baltic Sea using surface plasmon resonance," Environ. Sci. Technol. 47, 8582-8590 (2013).

[10] C. Delezoide, I. Ledoux-Rak, and C. T. Nguyen, “General approach for the sensitivity analysis and optimization of integrated optical evanescent-wave sensors," J. Opt. Soc. Am. B 31, 851-859 (2014).

[11] N. Daldosso, M. Melchiorri, F. Riboli, F. Sbrana, L. Pavesi, G. Pucker, C. Kompocholis, et al., "Fabrication and optical characterization of thin two-dimensional SizN4 waveguides," Mat. Sci. Semicon. Proc. 7, 453-458 (2004).

[12] S. M. Lindecrantz, and 0. G. Helles $\varnothing$, "Estimation of propagation losses for narrow strip and rib waveguides," IEEE Photon. Technol. L. 26, 1836-1839 (2014).

[13] X. Xu, S. Chen, J. Yu, and X. Tu, "An investigation of the mode characteristics of Sol submicron rib waveguides using the film mode matching method," J. Opt. A-Pure Appl. 0p. 11, 015508 (2009).

[14] F. T. Dullo, J.-C. Tinguely, S. A. Solb $\phi$, and 0. G. Helles $\phi$, "Singlemode limit and bending losses for shallow rib $\mathrm{Si}_{3} \mathrm{~N}_{4}$ waveguides," IEEE Photon. J. 7, 1-11 (2015).

[15] F. Prieto, B. Sepúlveda, A. Calle, A. Llobera, C. Domínguez, A. Abad, and A. Montoya, "An integrated optical interferometric nanodevice based on silicon technology for biosensor applications," Nanotechnology 14, 907-912 (2003).

[16] K. Tiefenthaler, and W. Lukosz, "Sensitivity of grating couplers as integrated-optical chemical sensors," J. Opt. Soc. Am. B 6, 209-220 (1989).

[17] F. Prieto, A. Llobera, D. Jiménez, C. Doménguez, A. Calle, and L. M. Lechuga, "Design and analysis of silicon antiresonant reflecting optical waveguides for evanscent field sensor," J. Lightwave Technol. 18, 966-972 (2000).

[18] G. L. Klunder, and R. E. Russo, "Core-based intrinsic fiber-optic absorption sensor for the detection of volatile organic compounds," Appl. Spectrosc. 49, 379-385 (1995).

[19] K. Zinoviev, L. G. Carrascosa, J. Sánchez del Río, B. Sepúlveda, C. Domínguez, and L. M. Lechuga, "Silicon photonic biosensors for lab-on-a-chip applications," Adv. Opt. Technol. 2008, 383927 (2008). 